\title{
Prognostic factors for candidaemia in intensive care unit patients: a retrospective analysis
}

\author{
Yasumasa Kawano ${ }^{1}$, MD, Atsushi $\underline{\text { Togawa }}^{2}$, MD, PhD, Yoshihiko Nakamura ${ }^{1}$, MD, Mariko Mizunuma ${ }^{1}$, MD, \\ Reiko Yamasaki ${ }^{1}$, MD, Kota Hoshino ${ }^{1}$, MD, Takeshi Nishida ${ }^{1}, \mathrm{MD}$, Hiroyasu Ishikura ${ }^{1}$, MD, PhD
}

INTRODUCTION Candidaemia, recognised as a fairly common disease among intensive care unit (ICU) patients, carries a poor prognosis. However, as studies on the prognostic factors associated with candidaemia in ICU patients are limited, this study aimed to establish the best prognostic factor for ICU patients with candidaemia in a tertiary care hospital in Japan. METHODS We conducted a retrospective cohort study of patients with candidaemia in the emergency ICU at Fukuoka University Hospital, Fukuoka, Japan, from April 2010 to March 2015. Demographic and clinical data was collected from the patients' medical records and laboratory databases.

RESULTS A total of 25 patients were included in the study. However, 18 patients died during hospitalisation, resulting in an in-hospital mortality rate of $72.0 \%$. The variables of Sequential Organ Failure Assessment (SOFA) score and cumulative number of risk factors for invasive candidiasis showed significant differences between patients in the survivor and nonsurvivor groups $(p<0.05)$. The areas under the receiver operating characteristic curves for the SOFA score and cumulative number of risk factors for invasive candidiasis were 0.873 (95\% confidence interval $[\mathrm{Cl}] 0.72-1.00)$ and $0.937(95 \% \mathrm{Cl}$ 0.84-1.00), respectively.

CONCLUSION Our results suggest that the cumulative number of risk factors for invasive candidiasis was the most useful prognostic indicator for candidaemia in ICU patients.

Keywords: candidaemia, intensive care unit, prognostic factor

\section{INTRODUCTION}

Invasive candidiasis, which is known to encompass candidaemia and deep-seated candidiasis (infections of tissue sites beneath the mucosal surface), ${ }^{(1)}$ is one of the most well-known nosocomial infections. ${ }^{(2)}$ Patients admitted to an intensive care unit (ICU) are at high risk for developing invasive candidiasis. ${ }^{(3)}$ It has proven difficult to precisely diagnose invasive candidiasis ${ }^{(4)}$ due to the low sensitivity (38\%) of blood cultures.(1) However, blood culture remains an important tool in the diagnosis of invasive candidiasis. ${ }^{(5)}$ According to recommendations from experts and learned societies, a single positive blood culture is sufficient to make a diagnosis of invasive candidiasis and justifies the initiation of antifungal treatment. ${ }^{(6)}$ Studies have reported that candidaemia makes up $10 \%$ of all bloodstream infections in the $\mathrm{ICU}^{(2)}$ and has a mortality rate of $30 \%-60 \% .^{(2,7-9)}$ It is, therefore, clearly a significant infection among ICU patients.

Several studies have reported on the prognostic factors associated with candidaemia in hospitalised patients..$^{(8-13)}$ These factors include: the use of mechanical ventilation; hypoproteinaemia; infections by Candida albicans (C. albicans) or Candida tropicalis (C. tropicalis); a higher Acute Physiology and Chronic Health Evaluation (APACHE) II score; delay in catheter withdrawal; and inadequate antifungal therapy. Although these factors are typically useful as prognostic factors for patients in the general ward, they may not be useful for ICU patients with critical status and severe morbidity. In these patient populations, a combination of prognostic factors may more accurately predict the clinical course of candidaemia rather than a single prognostic factor. Since the prognostic factors associated with candidaemia are very similar to the risk factors for invasive candidiasis, we speculated that the cumulative number of risk factors for invasive candidiasis could be a more useful prognostic indicator for candidaemia than the prognostic factors reported in previous studies. The objective of this study was to demonstrate this hypothesis among ICU patients.

\section{METHODS}

We conducted a retrospective study from April 2010 to March 2015 to investigate the prognostic factors associated with candidaemia among patients admitted to the emergency ICU at Fukuoka University Hospital, a 915-bed facility in Fukuoka, Japan. A case of candidaemia was defined as having at least one positive blood culture of Candida spp. Multiple episodes of candidaemia occurring in the same patient during the same hospital stay were counted as a single episode. Candida spp. were isolated from positive blood culture bottles (BacT/ALERT ${ }^{\circledR}$ SN; bioMérieux Inc, Marcy-l'Étoile, France) by subculturing separately on sheep blood agar and chocolate agar plates. The presence of Candida was evaluated using identification tests with CHROMagar Candida medium (Kanto Chemical Co Ltd, Tokyo, Japan) and VITEK 2 system (bioMérieux Inc, MarcyI'Étoile, France).

In this retrospective study, the endpoint was to discover the most useful prognostic factor for candidaemia. The patients' medical records were reviewed for potential prognostic factors

${ }^{1}$ Department of Emergency and Critical Care Medicine, Faculty of Medicine, ${ }^{2}$ Division of Medical Oncology, Hematology and Infectious Diseases, Fukuoka University, Japan Correspondence: Dr Yasumasa Kawano, Research Associate, Department of Emergency and Critical Care Medicine, Faculty of Medicine, Fukuoka University, 7-45-1 Nanakuma, Jonan-ku, Fukuoka 8140180, Japan. kawano0301@cis.fukuoka-u.ac.jp 
of candidaemia, including the identified species of Candida, Candida score, (1-3)-beta-D-glucan levels, blood lactate levels, C-reactive protein levels, Sequential Organ Failure Assessment (SOFA) score and the cumulative number of risk factors for invasive candidiasis. These factors, except the identified species of Candida, were evaluated within 24 hours of collection of the blood culture sample that became positive for Candida spp. The removal or exchange of a central venous catheter (CVC) and the initiation of antifungal therapy, which were evaluated within 48 hours of collection of blood culture, were also considered to be potential prognostic factors.

The Candida score $^{(14)}$ was designed to enable rapid recognition of Candida infections in order to initiate early antifungal therapy. It was calculated using four independent risk factors: multifocal Candida spp. colonisation; surgery upon ICU admission; severe sepsis; and total parenteral nutrition (TPN). (1-3)-beta-D-glucan levels were measured using a colourimetric assay (Wako Pure Chemical Industries Ltd, Chuo-Ku, Osaka, Japan), ${ }^{(15)}$ with a cut-off value of $7 \mathrm{pg} / \mathrm{mL}$, so that a (1-3)-beta-D-glucan level under the cut-off value was counted as $0 \mathrm{pg} / \mathrm{mL}$. The SOFA score, ${ }^{(16)}$ which estimates organ dysfunction related to various disease statuses, was calculated using readily available measurements to quantify the dysfunction of six major organs. The cumulative number of risk factors for invasive candidiasis was counted from a total number of 15 risk factors (Box 1). ${ }^{(3)}$ The APACHE II score, like the SOFA score, is well known as a severity score for critical patients, ${ }^{(17)}$ and has also been reported to be a prognostic factor associated with candidaemia in previous studies..$^{(8,9,11-13)}$ However, the APACHE II score was excluded from the present study, as it was already listed as a risk factor for invasive candidiasis (Box 1).

\begin{tabular}{|c|}
\hline 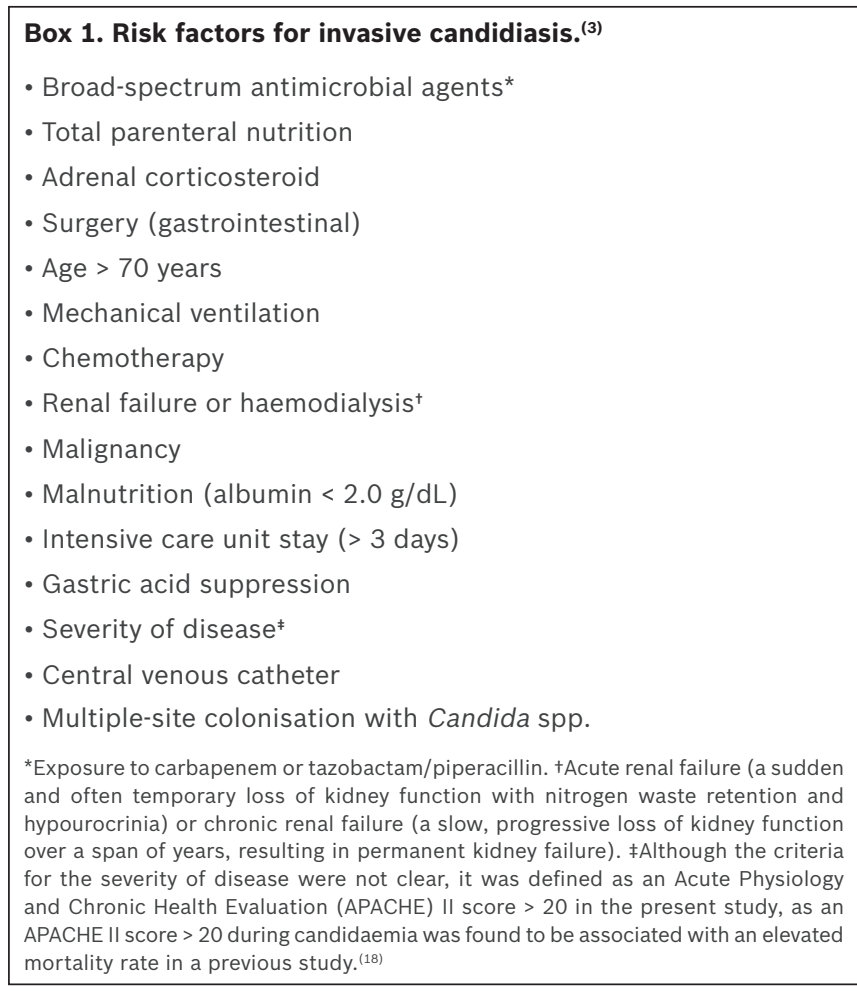 \\
\hline
\end{tabular}

Continuous data was expressed as median (range) or percentage values. Statistical differences between the two groups were determined using Mann-Whitney $U$ test and chisquare test, as appropriate. Receiver operating characteristic (ROC) analysis, including the area under the ROC curve (AUROC), was used to evaluate the prognostic value of the identified risk factors for candidaemia. The ROC curve was plotted for each score using sensitivity and specificity values for true prediction of ICU mortality across the entire range of potential cut-off values to predict mortality. The AUROC was constructed and compared, as described in a previous report. ${ }^{(19)}$ All tests were two-tailed and $p<0.05$ was considered to be statistically significant.

All statistical analyses were performed using the EZR software program (Easy R®; Saitama Medical Center, Jichi Medical University, Saitama, Japan), ${ }^{(20)}$ a graphical user interface for the R software program (The R Foundation for Statistical Computing, Vienna, Austria). The EZR software program is a modified version of the R Commander that was designed to add statistical functions frequently used in biostatistics.

\section{RESULTS}

A total of 4,136 patients were admitted to the ICU over the five-year study period. After excluding one patient with candidaemia who lacked laboratory data, a total of 25 patients with candidaemia were enrolled in the study. The incidence rate of candidaemia in our study was 62.9 per 10,000 admissions without prophylactic antifungal therapy.

The characteristics of the patients with candidaemia are shown in Table I. Out of 25 patients, 18 died during hospitalisation and seven survived, resulting in an in-hospital mortality rate of $72.0 \%$. The median age of the patients was 69 (range 24-88) years, and 14 (56.0\%) patients were male. Use of broad-spectrum antimicrobial agents $(84.0 \%)$, mechanical ventilation (72.0\%), gastric acid suppression (100.0\%) and an ICU stay $>3$ days $(76.0 \%)$ were observed in a majority of patients. Moreover, approximately half $(44.0 \%)$ of the patients received TPN. Conversely, no patient had neutropenia (absolute neutrophil count $<500$ cells $/ \mathrm{mm}^{3}$ ), few $(12.0 \%)$ patients had malnutrition (albumin $<2.0 \mathrm{~g} / \mathrm{dL}$ ) and only one (4.0\%) patient received adrenal corticosteroid therapy. The median length of ICU stay prior to the onset of candidaemia was 13 (range 1-73) days. Over half $(56.0 \%)$ of our patients were diagnosed with sepsis as the primary diagnosis on admission. The most frequent causes of sepsis in these patients were pneumonia $(21.4 \%)$ or skin and soft-tissue infections (21.4\%).

Candida spp. isolated from blood cultures taken from patients with candidaemia were compared between the survivors and non-survivors (Table II). Approximately half of all patients in the two groups had candidaemia due to $C$. albicans, and there was no significant difference in the composition of Candida spp. between the two patient groups. When the factors associated with in-hospital mortality were compared (Table III), the SOFA score and cumulative number of risk factors for invasive candidiasis were found to be significantly different between the two groups 
Table I. Baseline characteristics and main diagnosis of ICU patients with candidaemia $(n=25)$.

\begin{tabular}{|c|c|}
\hline Variable & $\begin{array}{c}\text { Median (range)/ } \\
\text { no. (\%) }\end{array}$ \\
\hline Age (yr) & $69(24-88)$ \\
\hline Male gender & $14(56.0)$ \\
\hline Broad-spectrum antimicrobial agents* & $21(84.0)$ \\
\hline Neutropenia $^{+}$ & $0(0)$ \\
\hline ICU stay (> 3 days) & $19(76.0)$ \\
\hline Adrenal corticosteroid & $1(4.0)$ \\
\hline Mechanical ventilation & $18(72.0)$ \\
\hline Haemodialysis & $15(60.0)$ \\
\hline Gastric acid suppression & $25(100.0)$ \\
\hline Malnutrition (albumin $<2.0 \mathrm{~g} / \mathrm{dL}$ ) & $3(12.0)$ \\
\hline Multiple-site colonisation with Candida spp. & $11(44.0)$ \\
\hline Total parenteral nutrition & $11(44.0)$ \\
\hline LOS in ICU prior to candidaemia (day) & $13(1-73)$ \\
\hline \multicolumn{2}{|l|}{ Main diagnosis on admission } \\
\hline Burn & $2(8.0)$ \\
\hline Sepsis & $14(56.0)$ \\
\hline Pneumonia & $3(21.4)$ \\
\hline Skin and soft-tissue infections & $3(21.4)$ \\
\hline Bacterial translocation & $2(14.3)$ \\
\hline Meningitis & $1(7.1)$ \\
\hline Lower gastrointestinal perforation & $1(7.1)$ \\
\hline Catheter-associated infection & $1(7.1)$ \\
\hline Other cause & $3(21.4)$ \\
\hline Acute drug intoxication & $1(4.0)$ \\
\hline Upper gastrointestinal haemorrhage & $1(4.0)$ \\
\hline Stroke & $1(4.0)$ \\
\hline Fulminant hepatitis & $1(4.0)$ \\
\hline Acute pancreatitis & $1(4.0)$ \\
\hline Toxic epidermal necrolysis & $1(4.0)$ \\
\hline Trauma & $1(4.0)$ \\
\hline Hypoxic-ischaemic brain injury & $1(4.0)$ \\
\hline Thoracoabdominal aortic aneurysm rupture & $1(4.0)$ \\
\hline
\end{tabular}

*Exposure to carbapenem or tazobactam/piperacillin. †Absolute neutrophil count $<500$ cells $/ \mathrm{mm}^{3}$. ICU: intensive care unit; LOS: length of stay

Table II. Candida spp. isolated from survivors and non-survivors.

\begin{tabular}{lccc}
\hline Candida spp. & \multicolumn{2}{c}{ No. (\%) } & p-value \\
\cline { 2 - 3 } & $\begin{array}{c}\text { Survivors } \\
(\mathbf{n}=\mathbf{7})\end{array}$ & $\begin{array}{c}\text { Non-survivors } \\
(\mathbf{n}=\mathbf{1 8})\end{array}$ & \\
\hline Candida albicans & $4(57.1)$ & $9(50.0)$ & 1.00 \\
Candida parapsilosis & $1(14.3)$ & $1(5.6)$ & 0.49 \\
Candida tropicalis & $2(28.6)$ & $1(5.6)$ & 0.18 \\
Candida glabrata & $0(0)$ & $5(27.8)$ & 0.27 \\
Candida lusitaniae & $0(0)$ & $2(11.1)$ & 1.00 \\
\hline
\end{tabular}

$(p<0.05)$. However, none of the other variables was found to be significantly different between the two groups.

The AUROC for the SOFA score was 0.873 (95\% confidence interval [CI] 0.72-1.00) and that for the cumulative number of risk factors for invasive candidiasis was 0.937 (95\% $\mathrm{Cl}$ 0.84-1.00) (Table IV). Between these two factors, the
Table III. Factors associated with in-hospital mortality among patients with candidaemia $(n=25)$.

\begin{tabular}{|c|c|c|c|}
\hline \multirow[t]{2}{*}{ Variable } & \multicolumn{2}{|c|}{ Median (range)/no. (\%) } & \multirow[t]{2}{*}{ p-value } \\
\hline & $\begin{array}{c}\text { Survivors } \\
(n=7)\end{array}$ & $\begin{array}{c}\text { Non-survivors } \\
(n=18)\end{array}$ & \\
\hline Candida score & $2(0-3)$ & $3(0-4)$ & 0.07 \\
\hline$B G(p g / m L)$ & $0(0-135)$ & $19.8(0-600)$ & 0.44 \\
\hline Lactate $(\mathrm{mg} / \mathrm{dL})$ & $16(8-25)$ & $19(8-139)$ & 0.25 \\
\hline $\begin{array}{l}\text { C-reactive } \\
\text { protein }(\mathrm{mg} / \mathrm{dL})\end{array}$ & $11.1(1.2-30)$ & $8.7(0.4-27.4)$ & 0.93 \\
\hline SOFA score & $6(2-12)$ & $12.5(1-23)$ & $<0.05^{\dagger}$ \\
\hline $\begin{array}{l}\text { Cumulative no. } \\
\text { of risk factors for } \\
\text { invasive candidiasis }\end{array}$ & $4(2-7)$ & $8(5-11)$ & $<0.05^{+}$ \\
\hline Presence of CVC & $2(28.6)$ & $14(77.8)$ & 0.06 \\
\hline $\begin{array}{l}\text { Removal/exchange } \\
\text { of } \mathrm{CVC}^{*}\end{array}$ & $2(100.0)$ & $8(57.1)$ & 0.50 \\
\hline $\begin{array}{l}\text { Start of antifungal } \\
\text { therapy* }\end{array}$ & $1(14.3)$ & $7(38.9)$ & 0.36 \\
\hline
\end{tabular}

*Evaluated within $48 \mathrm{hr}$ of collection of blood culture samples that were positive for Candida spp. $+p<0.05$ was statistically significant. BG: (1-3)-beta-D-glucan; CVC: central venous catheter; SOFA: sequential organ failure assessment

cumulative number of risk factors for invasive candidiasis had the larger AUROC.

\section{DISCUSSION}

In this retrospective study, the SOFA score and cumulative number of risk factors for invasive candidiasis were identified as variables that predict hospital death due to candidaemia among ICU patients. Furthermore, the cumulative number of risk factors for invasive candidiasis was the most useful factor. Epidemiological data on invasive candidiasis from the United States' National Center for Health Statistics showed that the risk factors associated with invasive candidiasis were well established and had not changed substantially over the last two decades. ${ }^{(3)}$ As these risk factors are also not expected to change greatly in the future, the cumulative number of risk factors for invasive candidiasis is a valuable measure for predicting the outcome of candidaemia.

The risk factors for invasive candidiasis that were originally evaluated for the diagnosis of invasive candidiasis included the Candida score. ${ }^{(14)}$ This score has a sensitivity and specificity of $77 \%$ and $66 \%$, respectively, for identifying patients with current or future invasive candidiasis. ${ }^{(21)}$ Few studies have reported the relationship between the Candida score and the prognostic factors of candidaemia, and the present study showed that there was no relationship. On the other hand, the cumulative number of risk factors for invasive candidiasis was found to be associated with prognostic factors for candidaemia among our ICU patients. These findings suggest that evaluating the risk factors for invasive candidiasis is a readily available method that can be employed when treating patients with candidaemia, especially with regard to diagnosis and outcome.

Previous reports have indicated the presence of $C$. albicans or $C$. tropicalis as independent adverse prognostic indicators. ${ }^{(8,10)}$ However, this was not clear in the present study, as the distribution 
Table IV. Mortality prediction in invasive candidiasis patients using receiver operating characteristic curves.

\begin{tabular}{|c|c|c|c|c|c|c|c|}
\hline Mortality predictor & AUROC & $95 \% \mathrm{Cl}$ & $\begin{array}{c}\text { Optimal } \\
\text { cut-off value }\end{array}$ & $\begin{array}{c}\text { Sensitivity } \\
\text { (\%) }\end{array}$ & $\begin{array}{c}\text { Specificity } \\
(\%)\end{array}$ & $\begin{array}{l}\text { PPV } \\
(\%)\end{array}$ & $\begin{array}{l}\text { NPV } \\
(\%)\end{array}$ \\
\hline SOFA score & 0.873 & $0.72-1.00$ & 6 & 71.4 & 94.4 & 81.0 & 75.0 \\
\hline
\end{tabular}

AUROC: area under receiver operating characteristic curve; CI: confidence interval; NPV: negative predictive value; PPV: positive predictive value; SOFA: sequential organ failure assessment

of these Candida spp. was not significantly different between patients in the survivor and non-survivor groups. (1-3)-beta-Dglucan, which is frequently used as a diagnostic tool of serum biomarkers for identifying patients with invasive candidiasis in Japan, has a sensitivity of $83 \%$ and specificity of $86 \%$. ${ }^{(22)}$ However, $48.0 \%(12 / 25)$ of patients with candidaemia in our study were (1-3)-beta-D-glucan-negative. Koo et al demonstrated that the initial values of (1-3)-beta-D-glucan were not predictive of mortality or clinical outcome, ${ }^{(23)}$ and our results are in accordance with their study.

The incidence of candidaemia in our study was 62.9 per 10,000 admissions. Our results indicate a relatively high rate when compared with recent studies that reported a rate of 3.06 per 10,000 admissions in an Italian general ward ${ }^{(18)}$ and 15.1 per 10,000 admissions in a Chinese ICU population. ${ }^{(24)}$ This variability in the incidence rates of candidaemia among different countries may be reflective of differences in their healthcare systems, patient backgrounds and/or surveillance methods.

The in-hospital mortality rate in our study was $72.0 \%$ (18/25 patients), which was also higher than that observed in previous studies, ${ }^{(2,7-9)}$ especially a 2015 Japanese study $(26.5 \%) .{ }^{(25)}$ There are three possible reasons for the higher mortality rate seen among our patients. First, our study included many severely ill patients (mean APACHE II score 20.5, mean SOFA score 11.2). Second, antifungal drug treatment was started within 48 hours of collection of blood culture sample for only a few of our patients $(n=8 ; 32.0 \%)$. Although the ideal time for starting antifungal drugs is not yet known, previous studies have found correlations between worse prognosis and delays in the initiation of antifungal therapy and inadequate empirical antifungal therapy. ${ }^{(11-13,26,27)}$ These studies and our findings suggest that early antifungal administration is key. Lastly, the number of patients (10/16 patients, 62.5\%) for whom CVC was removed or exchanged within 48 hours of collection of blood culture sample was too low to have reduced hospital death in our study. This is important, as numerous studies have demonstrated that catheter removal is important to lower mortality that is associated with candidaemia. ${ }^{(8,11,26)}$

There are some limitations associated with the present study. As a retrospective study, it involved a single centre and had a small sample population. Also, we were unable to identify the unknown risk factors for invasive candidiasis (e.g. new biomarkers), possible contamination of blood cultures, the adequate type and dosage of antifungal agents for treatment, and the weighting of each risk factor for invasive candidiasis.

In conclusion, we have demonstrated that the cumulative number of risk factors for invasive candidiasis was the most useful prognostic indicator for candidaemia in ICU patients. This variable ought to be utilised as a means of validating the timing of initiating antifungal therapy to decrease in-hospital mortality.

\section{ACKNOWLEDGEMENT}

We sincerely thank Ms Kanae Misumi from the Department of Emergency and Critical Care Medicine, Faculty of Medicine, Fukuoka University, Fukuoka, Japan, for her help with data encoding.

\section{REFERENCES}

1. Clancy CJ, Nguyen $\mathrm{MH}$. Finding the "missing $50 \%$ " of invasive candidiasis: how nonculture diagnostics will improve understanding of disease spectrum and transform patient care. Clin Infect Dis 2013; 56:1284-92.

2. Wisplinghoff $H$, Bischoff $T$, Tallent SM, et al. Nosocomial bloodstream infections in US hospitals: analysis of 24,179 cases from a prospective nationwide surveillance study. Clin Infect Dis 2004; 39:309-17.

3. Pfaller MA, Diekema DJ. Epidemiology of invasive candidiasis: a persistent public health problem. Clin Microbiol Rev 2007; 20:133-63.

4. Blot S, Vandewoude K. Management of invasive candidiasis in critically ill patients. Drugs 2004; 64:2159-75.

5. Richardson MD, Carlson P. Culture and non-culture based diagnostics for Candida species. In: Calderone R, eds. Candida and candidosis. Washington: ASM Press, 2002: 387-425.

6. Edwards JE Jr, Bodey GP, Bowden RA, et al. International Conference for the Development of a Consensus on the Management and Prevention of Severe Candidal Infections. Clin Infect Dis 1997; 25:43-59.

7. Jarvis WR. Epidemiology of nosocomial fungal infections, with emphasis on Candida species. Clin Infect Dis 1995; 20:1526-30.

8. Andes DR, Safdar N, Baddley JW, et al; Mycoses Study Group. Impact of treatment strategy on outcomes in patients with candidemia and other forms of invasive candidiasis: a patient-level quantitative review of randomized trials. Clin Infect Dis 2012; 54:1110-22.

9. Fraser $\mathrm{VJ}$, Jones $\mathrm{M}$, Dunkel J, et al. Candidemia in a tertiary care hospital: epidemiology, risk factors, and predictors of mortality. Clin Infect Dis 1992; 15:414-21.

10. Zhang XB, Yu SJ, Yu JX, et al. Retrospective analysis of epidemiology and prognostic factors for candidemia at a hospital in China, 2000-2009. Jpn J Infect Dis 2012; 65:510-5.

11. Garnacho-Montero J, Díaz-Martín A, García-Cabrera E, et al. Impact on hospital mortality of catheter removal and adequate antifungal therapy in Candida spp. bloodstream infections. J Antimicrob Chemother 2013; 68:206-13.

12. Bassetti M, Righi E, Ansaldi F, et al. A multicenter study of septic shock due to candidemia: outcomes and predictors of mortality. Intensive Care Med 2014; 40:839-45

13. Garey KW, Rege M, Pai MP, et al. Time to initiation of fluconazole therapy impacts mortality in patients with candidemia: a multi-institutional study. Clin Infect Dis 2006; 43:25-31.

14. León C, Ruiz-Santana S, Saavedra P, et al; EPCAN Study Group. A bedside scoring system ("Candida score") for early antifungal treatment in nonneutropenic critically ill patients with Candida colonization. Crit Care Med 2006; 34:730-7.

15. Moro $\mathrm{H}$, Tsukada $\mathrm{H}$, Ohara $\mathrm{T}$, et al. [Clinical evaluation of performance of a new diagnostic method for deep mycosis by measuring beta-glucan concentration in the blood]. Kansenshogaku Zasshi 2003; 77:219-26. Japanese.

16. Ferreira FL, Bota DP, Bross A, Mélot C, Vincent IL. Serial evaluation of the SOFA score to predict outcome in critically ill patients. JAMA 2001; 286:1754-8 
17. Knaus WA, Draper EA, Wagner DP, Zimmerman JE. APACHE II: a severity of disease classification system. Crit Care Med 1985; 13:818-29.

18. Caggiano G, Coretti C, Bartolomeo N, et al. Candidabloodstream infections in Italy: changing epidemiology during 16 years of surveillance. Biomed Res Int 2015; 2015:256580.

19. Hanley JA, McNeil BJ. The meaning and use of the area under a receiver operating characteristic (ROC) curve. Radiology 1982; 143:29-36.

20. Kanda Y. Investigation of the freely available easy-to-use software 'EZR' for medical statistics. Bone Marrow Transplant 2013; 48:452-8.

21. León C, Ruiz-Santana S, Saavedra P, et al; Cava Study Group. Usefulness of the "Candida score" for discriminating between Candida colonization and invasive candidiasis in non-neutropenic critically ill patients: a prospective multicenter study. Crit Care Med 2009; 37:1624-33.

22. Moro H, Tsukada H, Ohara T, et al. [Comparison of four diagnostic methods using clinical blood by measuring (1-->3)-beta-D-glucan]. Kansenshogaku Zasshi 2003; 77:227-34. Japanese.

23. Koo S, Baden LR, Marty FM. Post-diagnostic kinetics of the (1 --> 3)- $\beta$-D glucan assay in invasive aspergillosis, invasive candidiasis and Pneumocystis jirovecii pneumonia. Clin Microbiol Infect 2012; 18:E122-7.

24. Li C, Wang H, Yin M, et al. The differences in the epidemiology and predictors of death between candidemia acquired in intensive care units and other hospital settings. Intern Med 2015; 54:3009-16.

25. Takesue Y, Ueda T, Mikamo H, et al; ACTIONs Project. Management bundles for candidaemia: the impact of compliance on clinical outcomes. J Antimicrob Chemother 2015; 70:587-93

26. Puig-Asensio M, Pemán J, Zaragoza R, et al; Prospective Population Study on Candidemia in Spain (CANDIPOP) Project; Hospital Infection Study Group (GEIH); Medical Mycology Study Group (GEMICOMED) of the Spanish Society of Infectious Diseases and Clinical Microbiology (SEIMC); Spanish Network for Research in Infectious Diseases. Impact of therapeutic strategies on the prognosis of candidemia in the ICU. Crit Care Med 2014; 42:1423-32.

27. Nolla-Salas J, Sitges-Serra A, León-Gil C, et al. Candidemia in nonneutropenic critically ill patients: analysis of prognostic factors and assessment of systemic antifungal therapy. Study Group of Fungal Infection in the ICU. Intensive Care Med 1997; 23:23-30. 